\title{
Altering the Biodegradation of Mesoporous Silica Nanoparticles by Means of Experimental Parameters and Surface Functionalization
}

\author{
Stephanie Seré (D), ${ }^{1}$ Bert De Roo, ${ }^{1}$ Mattias Vervaele, ${ }^{1}$ Stefaan Van Gool, ${ }^{2}$ Sandra Jacobs, ${ }^{3}$ \\ Jin Won Seo, ${ }^{4}$ and Jean-Pierre Locquet $\mathbb{D}^{1}$
}

${ }^{1}$ Department of Physics and Astronomy, KU Leuven, Laboratory for Semiconductor Physics, 3001 Leuven, Belgium

${ }^{2}$ Immunologisch Onkologisches Zentrum Köln, 50674 Köln, Germany

${ }^{3}$ Department of Microbiology and Immunology, KU Leuven, 3000 Leuven, Belgium

${ }^{4}$ Department of Materials Engineering, KU Leuven, 3001 Leuven, Belgium

Correspondence should be addressed to Stephanie Seré; stephanie.sere@kuleuven.be and Jean-Pierre Locquet; jeanpierre.locquet@kuleuven.be

Received 6 September 2017; Revised 10 January 2018; Accepted 30 January 2018; Published 7 March 2018

Academic Editor: Swadeshmukul Santra

Copyright (C) 2018 Stephanie Seré et al. This is an open access article distributed under the Creative Commons Attribution License, which permits unrestricted use, distribution, and reproduction in any medium, provided the original work is properly cited.

\begin{abstract}
Mesoporous silica nanoparticles (MSNPs) are gaining a large interest in the field of medical and biomedical applications due to their biodegradability and high loading capacity as a carrier. In this work, a simple synthesis and functionalization procedure is reported, which allows tuning the nanoparticle properties, functionalization, and biodegradability. Variations in the synthesis procedure are introduced, including temperature, concentration of catalyst, and surface functionalization. These samples are characterized and afterwards degraded in phosphate buffered saline (PBS) to determine their degradation kinetics. The amount of degraded material is colorimetrically determined, using an optimized protocol based on molybdenum blue chemistry. It is shown that the degradability of the nanoparticles increased with decreasing synthesis temperatures, lower amounts of catalyst, and higher concentrations of nanoparticles. Surface functionalization alters the degradation kinetics as well, rendering amino-functionalized nanoparticles the fastest degradation behavior, followed by carboxylated and nonfunctionalized nanoparticles. From these results, it can be concluded that the degradation rate of MSNPs can be varied from a few hours to several days by small changes in the synthesis procedure. Moreover, the degradation behavior is strongly dependent on the nanoparticle growth rate.
\end{abstract}

\section{Introduction}

Research on MSNPs was launched in the 1990s, and the application of these nanoparticles (NPs) has been widely investigated ever since [1]. This profound interest arose from their advantageous physicochemical properties. MSNPs have high mechanical and chemical stability and abundant silanol groups facilitating functionalization. Additionally, they provide a large surface to volume ratio for functionalization, as well as adjustable pores and loading capacity for various molecules and substances. Being biocompatible and biodegradable whilst providing higher stability and more versatile immobilization compared to other carriers, MSNPs have especially high potential in biomedical applications, like drug delivery and bioimaging. Nonetheless, the use of MSNPs is being investigated in other scientific fields as well, such as catalysis and sensing [2-5]. For these applications, it is key for MSNPs to be sufficiently stable. As for biomedical applications, NP accumulation in vivo should be avoided and in this regard, MSNPs should degrade in biological media. Although the degradation kinetics of these MSNPs is very important, it has not been thoroughly examined and the effects of experimental parameters during synthesis are not yet entirely understood.

One of the first studies regarding the biodegradation kinetics of MSNPs was performed by $\mathrm{He}$ et al. [6]. They reported a three-stage degradation process of MSNPs in simulated body fluid (SBF), consisting of a fast degradation 
(stage 1), followed by a fast decrease in degradation on hour scale (stage 2), and finally a maintained slow degradation on day scale (stage 3) [6]. This three-step degradation behavior was not observed for nonporous silica NPs. Moreover, it was found that the method for template removal after synthesis influenced the degradation behavior of the MSNPs. Calcined NPs showed lower degradation percentages compared to NPs refluxed in hydrochloric acid [6].

An important parameter determining the biodegradability of silica NPs is their specific surface area. For nonporous silica NPs, the biodegradability is therefore directly proportional to the particle size, as shown by Yamada et al. [7]. The smaller the NPs, the greater the contact with the surrounding medium at the interface and the more degradable the NPs. Unfortunately, such small particles cause higher cell toxicity and hemolytic activity [8-11]. In contrast, the degradation of MSNPs is not size dependent in a size range of 20 to $80 \mathrm{~nm}$ diameter, as it is mainly caused by the enlarged surface area due to the pores [7]. This allows the use of larger, less toxic NPs with good biodegradability.

Besides surface area, the biodegradability of MSNPs is affected by surface functionalization as well. In the work published by Lin et al., the influence of a hydrophilic polymer shell (poly(ethylene glycol), PEG) was investigated by incubating the MSNPs in PBS and performing a silicomolybdic blue assay. It was found that nonpegylated MSNPs had greater degradation than pegylated MSNPs [12]. Moreover, Cauda et al. reported that longer and denser PEG shells resulted in slower biodegradation kinetics [13]. In another work, Cauda et al. investigated the degradation behavior of MSNPs with different functional groups, including phenyl-, chloropropyl, and aminopropyl- functionalizations. It was found that phenyl functionalized MSNPs degraded significantly faster than those with other functionalizations, followed by chloropropyl and aminopropyl functionalized MSNPs, respectively [14].

Finally, Hon et al. reported tailoring the biodegradability of MSNPs by thermal oxidation and silica coating. These NPs degraded after only 40 minutes, but the degradation was slowed down up to 3 hours after thermal oxidation [15].

In this work, we show that the biodegradation rate of the MSNPs can be tuned, by varying synthesis parameters such as temperature, base concentration, and surface functionalization of the MSNPs. Different degradation kinetics might be specifically interesting in biomedical applications. Delayed biodegradation may result in slow and controlled drug delivery, whereas fast degradation might be particularly interesting in applications with short half-life drugs.

\section{Experimental Section}

To investigate the influence of different experimental parameters on the biodegradability of the MSNPs, variations in the standard synthesis protocol were introduced. Both the temperature and the concentration of catalytic base were varied. Additionally, the influence of different ratios of MSNPs on the biological medium was examined. Finally, the biodegradability was tested for MSNPs with different functionalization, including bare MSNPs with silanol groups, amine-functionalized MSNPs, and carboxylated MSNPs.

2.1. MSNP Synthesis and Characterization. The synthesis protocol of MSNPs was based on the protocol of Yamada et al. [7] and is illustrated in Figure 1. $0.4 \mathrm{~g}$ triethanolamine (TEA, Acros Organics) and $0.5 \mathrm{~g}$ hexadecyltrimethylammonium bromide (CTAB, Acros Organics) were dissolved in $60 \mathrm{~mL}$ ultrapure water and stirred at $80^{\circ} \mathrm{C}$. In this research, the amount of TEA was varied from $0.2 \mathrm{~g}$ to $0.8 \mathrm{~g}$, and the temperature was varied in a range of $65^{\circ} \mathrm{C}$ to $80^{\circ} \mathrm{C}$. Thereafter, $0.491 \mathrm{~mL}$ tetraethoxysilane (TEOS, Sigma-Aldrich) was added and the solution was vigorously stirred. In order to render the MSNPs a carboxylic or amino-functionalization, 30 $\mu \mathrm{L}$ TEOS and $30 \mu \mathrm{L}$ carboxyethylsilanetriol (CES, Gelest) or (3-aminopropyl) triethoxysilane (APTES, Sigma-Aldrich), respectively, were added after 17 hours (Figures 1(b) and $1(\mathrm{c}))$. After stirring the mixture at $80^{\circ} \mathrm{C}$ for 24 hours in total, the MSNPs were dialyzed (dialysis tubing membrane $14 \mathrm{kDa}$ molecular weight cut-off (MWCO), Sigma-Aldrich) against a continuously stirred mixture of $280 \mathrm{~mL} 2 \mathrm{M}$ aqueous acetic acid solution (Sigma-Aldrich) and $280 \mathrm{~mL}$ ethanol for 12 hours to wash away the template. The mixture was refreshed 5 times. Subsequently, the membrane was brought into ultrapure water for 12 hours and refreshed 5 times to wash away the acetic acid. Finally, the MSNPs were characterized with different characterization techniques. Dynamic light scattering (DLS, VASCO particle size analyzer DL135, Cordouan Technologies) was performed at $25^{\circ} \mathrm{C}$ to determine the hydrodynamic diameter $\left(D_{\mathrm{H}}\right)$ of the MSNPs. The core diameter $\left(D_{\mathrm{C}}\right)$ was determined with transmission electron microscopy (TEM, CM200 FEG, Philips). For the TEM sample preparation, 8 to $10 \mu \mathrm{L}$ of NP solution was placed on a holey carbon-coated copper grid and dried under ambient conditions. At least $100 \mathrm{NPs}$ were analyzed with ImageJ to determine the mean core diameter [16]. Finally, surface coating and long-term stability data were obtained with zeta potential measurements (WALLIS zeta potential analyzer, Cordouan Technologies).

2.2. Biodegradation of MSNPs. $100 \mathrm{~mL}$ of $0.01 \mathrm{M}$ PBS (SigmaAldrich) was used as a model for biodegradation in the human body. The phosphates of PBS interact with the first layer of silicon atoms at the surface of the nanoparticles, increasing their coordination to more than fourfold as schematically represented in Figure 2. This in turn weakens the oxide bonds between the first and second layer of silicon atoms, favoring hydrolysis of silicon atoms and causing the MSNPs to degrade gradually from their outer surfaces [17]. Three different volume to volume ratios of MSNPs to PBS were used in this work: $2: 100,4: 100$, and $6: 100$. As shown in Figure 3, the synthesized MSNPs were dialyzed against PBS (dialysis tubing membrane $7 \mathrm{kDa}$ MWCO, Sigma-Aldrich). Every hour, a sample of PBS was taken and the PBS solution was refreshed to avoid a silicate $\left(\mathrm{Si}(\mathrm{OH})_{4}\right)$ concentration build-up.

2.3. Spectrophotometric Determination of Silicate with Molybdenum Blue. The amount of silicate in the PBS samples 


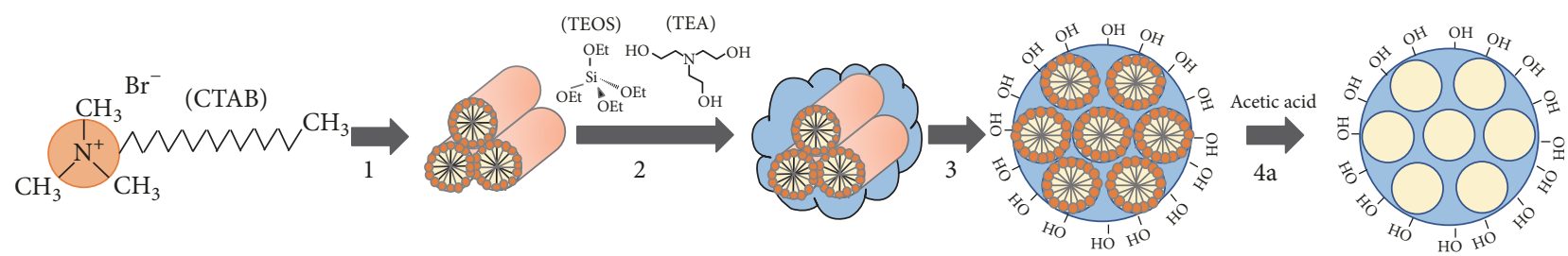

(a)

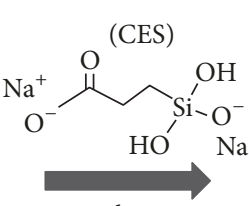

$4 \mathrm{~b}$

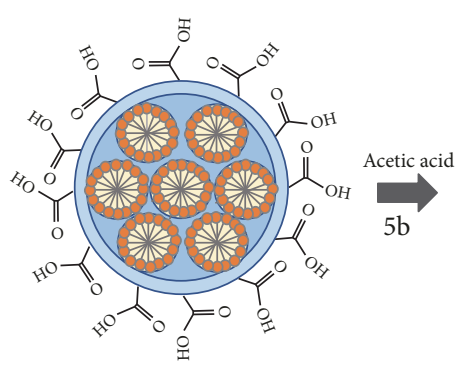

(b)

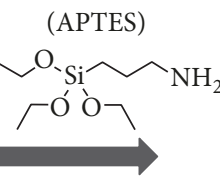

$4 c$
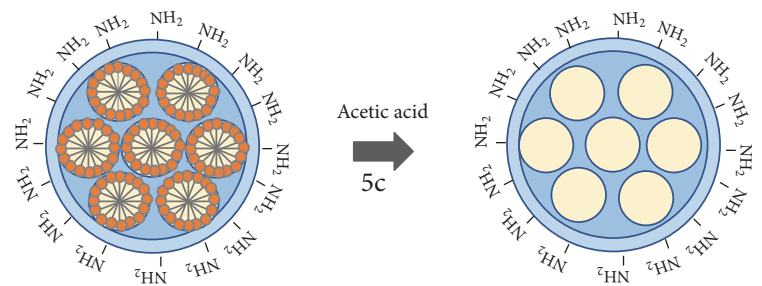

(c)

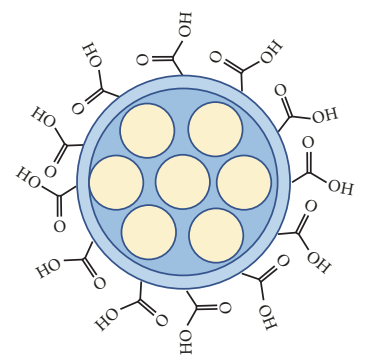

FIGURE 1: Synthesis protocol of (a) bare MSNPs, (b) carboxylated MSNPs, and (c) amino-functionalized MSNPs. In step 1, CTAB is dissolved in water to self-assemble into cylindrical micelles. In step 2, TEOS and TEA are added to form the NP. For NPs with silanol functionalization, the particles are reacted for 24 hours in step 3, after which the complete NPs are formed. For functionalized particles, step 3 includes the reaction of 17 hours, after which (step 4) the functionalization molecules are added. Finally, in step 5, the toxic CTAB is washed out of the pores, eventually resulting in (5a) bare MSNPs with silanol groups, (5b) carboxylated MSNPs, and (5c) amino-functionalized MSNPs.

was determined using molybdenum blue chemistry [18, 19], which is illustrated in Figure 4. First, the extinction of the samples was optimized by adding $0.3 \mathrm{~mL}$ of $1 \mathrm{M}$ hydrochloric acid $(V W R)$ to $1 \mathrm{~mL}$ sample. It was shown that the acidity of the sample influences the compound formed after further addition of molybdenum [20-22]. The $\alpha$-silicomolybdate compound has a lower extinction, as compared to $\beta$ silicomolybdate [20-22]. After assessing a range of different concentrations of hydrochloric acid, it was found that $0.3 \mathrm{~mL}$ of $1 \mathrm{M}$ hydrochloric acid was the optimal concentration for the amount of molybdate used in this work, following (1). Before adding molybdate, $1 \mathrm{~mL}$ ethanol (VWR) was added to stabilize the $\beta$-silicomolybdate compound [18]. Next, $1.5 \mathrm{~mL}$ of 5\% ammonium molybdate tetrahydrate (Acros Organics) was added to each PBS sample, producing a yellow color following (2a).

Besides forming silicomolybdic acid, molybdenum will react with phosphate ions from the PBS solution as well to form phosphomolybdic acid following (2b). In order to eliminate the influence of PBS on the absorption, $1 \mathrm{~mL}$ of $1 \mathrm{M}$ oxalic acid (Acros Organics) was added to each sample after 20 minutes. This induces a ligand exchange reaction, producing phosphate and molybdo-oxalate following (3).
Molybdosilicate does not undergo this reaction [23]. After reacting the sample 10 minutes with oxalic acid, $0.5 \mathrm{~mL}$ of $2 \%$ ascorbic acid (Acros Organics) was added to reduce the silicomolybdic acid to the molybdenum blue complex. Compared to the yellow silicomolybdic acid, the molybdenum blue complex has a higher molar extinction coefficient and therefore a better limit of detection [19]. After another 10 minutes, the absorption of the samples was measured at $810 \mathrm{~nm}$ (Ultrospec 2100 pro, amersham biosciences).

$$
\begin{aligned}
& \left(\mathrm{NH}_{4}\right)_{6} \mathrm{Mo}_{7} \mathrm{O}_{24} \cdot 4 \mathrm{H}_{2} \mathrm{O}+6 \mathrm{HCl} \\
& \Longleftrightarrow \mathrm{H}_{6} \mathrm{Mo}_{7} \mathrm{O}_{24} \cdot 4 \mathrm{H}_{2} \mathrm{O}+6 \mathrm{ClNH}_{4} \\
& 7 \mathrm{Si}(\mathrm{OH})_{4}+12 \mathrm{H}_{6} \mathrm{Mo}_{7} \mathrm{O}_{24} \cdot 4 \mathrm{H}_{2} \mathrm{O}+17 \mathrm{H}_{2} \mathrm{O} \\
& \Longleftrightarrow 7 \mathrm{H}_{4} \mathrm{SiMo}_{12} \mathrm{O}_{40} \cdot 29 \mathrm{H}_{2} \mathrm{O} \\
& 7\left(\mathrm{PO}_{4}\right)^{3-}+12 \mathrm{H}_{6} \mathrm{Mo}_{7} \mathrm{O}_{24} \cdot 4 \mathrm{H}_{2} \mathrm{O} \\
& \Longleftrightarrow 7 \mathrm{P}\left(\mathrm{Mo}_{12} \mathrm{O}_{40}\right)^{3-}+84 \mathrm{H}_{2} \mathrm{O} \\
& \mathrm{P}\left(\mathrm{Mo}_{12} \mathrm{O}_{40}\right)^{3-}+\mathrm{H}_{2} \mathrm{C}_{2} \mathrm{O}_{4} \\
& \Longleftrightarrow \mathrm{C}_{2}\left(\mathrm{Mo}_{12} \mathrm{O}_{40}\right)^{2-}+\mathrm{PO}_{3}{ }^{1-}+\mathrm{H}_{2} \mathrm{O}
\end{aligned}
$$




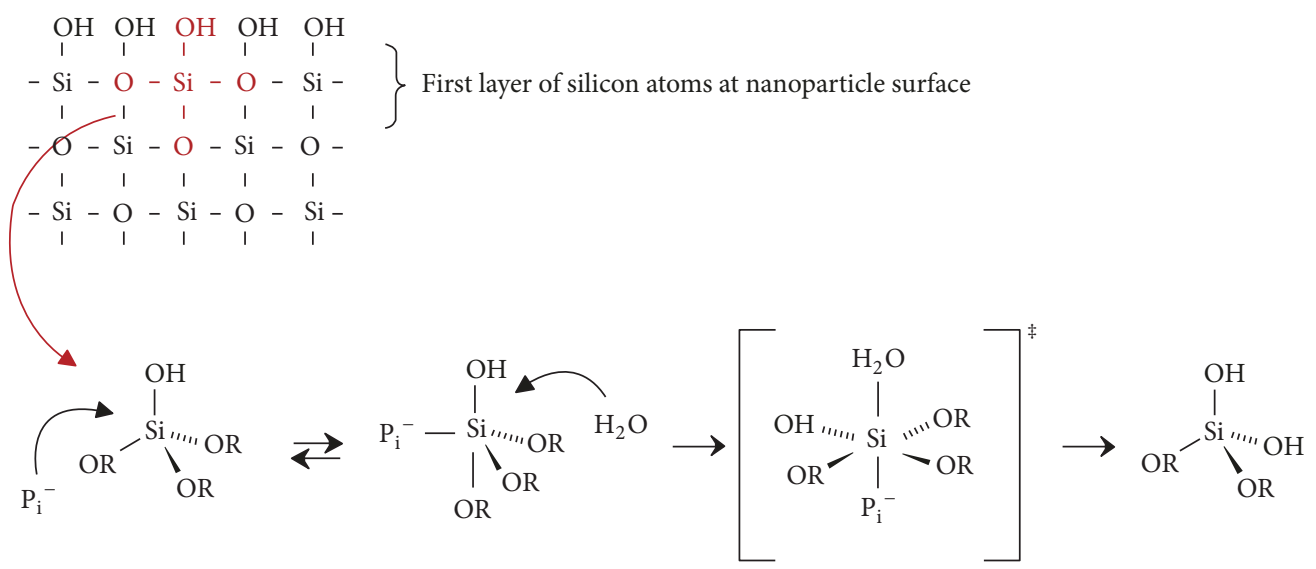

FIGURE 2: Schematic representation of the biodegradation chemistry. $\mathrm{P}_{\mathrm{i}}$ represents inorganic phosphates which are in physiological pH mostly $\mathrm{H}_{2} \mathrm{PO}_{4}{ }^{-}$. The coordination of the silicon atom increases to more than fourfold in the presence of a nucleophile such as $\mathrm{P}_{\mathrm{i}}$. This weakens the oxide bonds with the next layer of silicon atoms and favors hydrolysis.

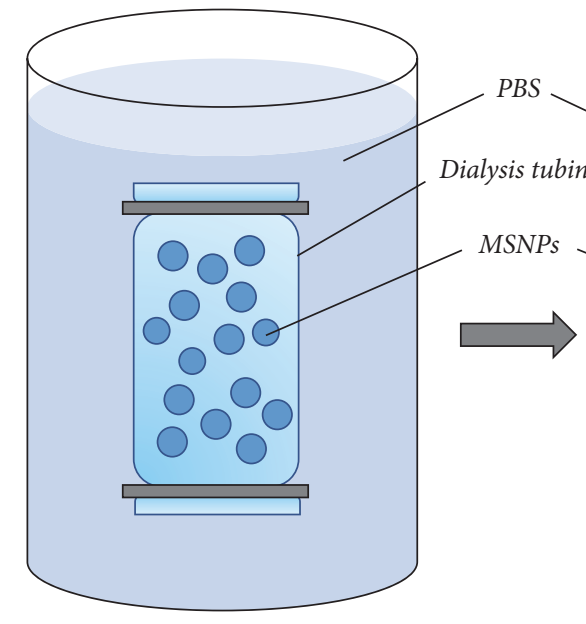

(a)

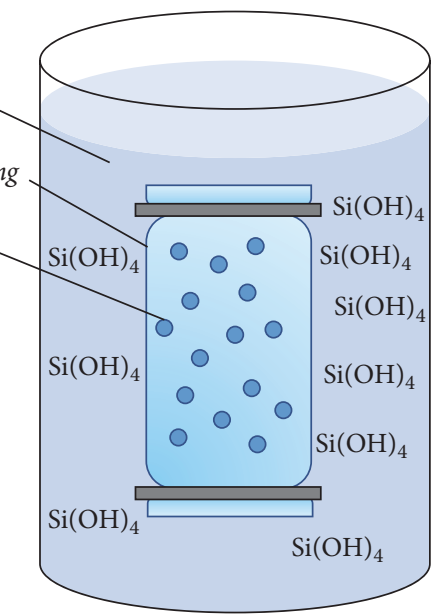

(b)

FIGURE 3: Schematic representation of MSNP dialysis against PBS. (a) represents the NP before starting degradation. (b) represent NPs that are partially degraded.

The calibration curve was set up, using standard solutions of silica (mono element standard $\mathrm{SiO} 2$, Servochem) and performing the same sample preparation and measurements explained in Figure 4. As this work evaluates the effect of the nanoparticle amount against PBS, among others, all results were normalized to the total amount of degraded material, which allows comparison of the different conditions. In this regard, when comparing the degradation kinetics, it should not be referred to as the absolute degradation kinetics but as the kinetics of the degraded material divided by the degraded material in total (i.e., the relative degradation speed is compared, not the absolute amount of material per unit of time).

\section{Results and Discussion}

3.1. MSNP Characterization. Different synthesis paths based on temperature changes, concentration of base catalyst, and surface functionalization were examined to understand their effect on the biodegradability of MSNPs. The physicochemical properties of these NPs are summarized in Table 1. From the TEM results, a steady increase in particle size was observed when more TEA was used in the reaction mixture or when the reaction temperatures were elevated. These findings are similar compared to those of Möller et al. [24] and are both attributed to the properties of TEA. At lower temperatures, TEA primarily acts as a base, leading to fast hydrolysis and condensation of TEOS, and hence the formation of small MNSPs. At higher temperatures, TEA is more likely to act as a chelating agent. Chelation of TEOS causes a decreased availability of silica precursor for the formation of mesoporous seeds and, therefore, formation of larger MSNPs [24]. Typical TEM images are given in Figure 5.

The same reasoning goes for the influence of the amount of TEA. When a large amount of TEA is used during synthesis of MSNPs at $80^{\circ} \mathrm{C}$, less TEOS will be available for rapid 

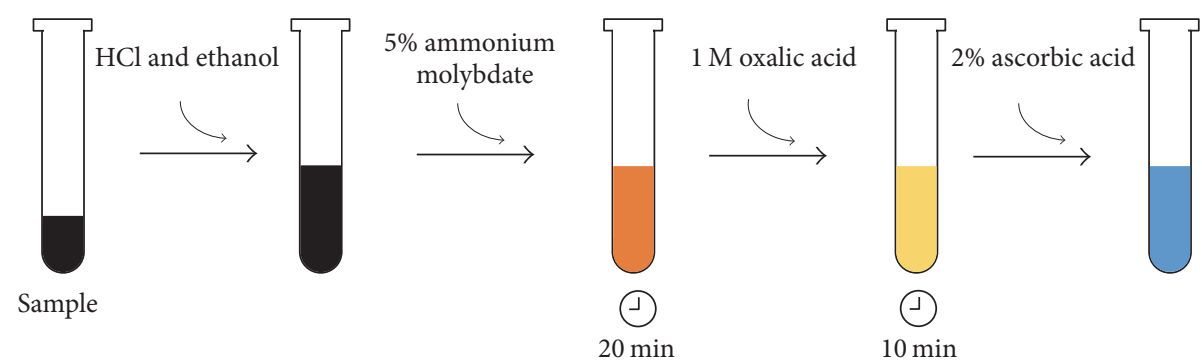

Figure 4: Chemical scheme of the molybdenum blue sample preparation.

TABLE 1: Physicochemical properties of different MSNPs.

\begin{tabular}{|c|c|c|c|c|c|}
\hline Sample & TEA [g] & $T\left[{ }^{\circ} \mathrm{C}\right]$ & $D_{\mathrm{H}}[\mathrm{nm}]$ & $D_{\mathrm{C}}[\mathrm{nm}]$ & $\zeta$-potential $[\mathrm{mV}]$ \\
\hline MSNP-OH-1 & 0.4 & 65 & 25.7 & $16.3 \pm 0.3$ & $-9.2 \pm 0.8$ \\
\hline MSNP-OH-2 & 0.4 & 80 & 29.5 & $17.8 \pm 0.3$ & $-8 \pm 1$ \\
\hline MSNP-OH-3 & 0.2 & 80 & 23.4 & $15.3 \pm 0.4$ & $-25 \pm 2$ \\
\hline MSNP-OH-4 & 0.8 & 80 & 20.4 & $20.2 \pm 0.3$ & $-14.2 \pm 0.8$ \\
\hline MSNP-OH-2 & 0.4 & 80 & 29.5 & $17.8 \pm 0.3$ & $-8 \pm 1$ \\
\hline $\mathrm{MSNP} \mathrm{NH}_{2}$ & 0.4 & 80 & 11.2 & & $21 \pm 2$ \\
\hline MSNP-COOH & 0.4 & 80 & 19.5 & $20.3 \pm 0.5$ & $-23 \pm 2$ \\
\hline
\end{tabular}

hydrolysis and condensation. This in turn leads to a low concentration of seeds and the formation of larger MSNPs. Lower particle sizes are obtained when less TEA is used and larger amounts of hydrolyzable TEOS remain available [24].

Finally, surface functionalization influenced the NP morphology as well. It was not possible to determine the core diameter of amine functionalized MSNPs with TEM, as they were too small and packed too dense together for analysis. For this reason, the hydrodynamic diameter obtained from DLS measurements was considered. Note that this diameter is often an overestimation due to the influence of surface coating and hydration layer on the diffusion of the NPs. Nevertheless, the DLS results suggest the size of aminefunctionalized NPs to be indeed much smaller compared to all other produced NPs. Additionally, from the zeta potential data, it is clear that the surface functionalization induced a higher NP stability compared to silanol coated NPs. Moreover, a clear indication of the surface coating was observed as the amine-functionalized NPs displayed a positive zeta potential and carboxylated MSNPs a negative zeta potential.

3.2. Biodegradability of MSNPs. In order to get an understanding about the structural evolution of the MSNPs during degradation, the degrading particles were visualized with TEM. Figure 6 represents MSNPs before degradation, eight hours and twenty-four hours after immersion in PBS from (a) to (c). Before degradation, single NPs can be distinguished and their pores are clearly visible. After eight hours, single NP identification becomes more difficult. The particles appear smaller but their porous structure remains, indicating that the particles degrade from their surfaces (i.e., the pores as well as the outer surface). In the TEM image after twenty-four hours, the particles have lost most of their structure. Neither pores nor single particles could be observed; however, a silicon matrix remains visible. After one week, the TEM images did not show any particles anymore (data not included).

The degradation behaviors of bare MSNPs, produced at $65^{\circ} \mathrm{C}$ (top curve) and $80^{\circ} \mathrm{C}$ (bottom curve), are illustrated in Figure 7. Both curves have the same shape. After a first, fast linear degradation step up to 26 hours, a decrease in degradation rate was observed for NPs synthesized at $65^{\circ} \mathrm{C}$. By comparison, the degradation curve of MSNPs produced at $80^{\circ} \mathrm{C}$ is shifted downward: that is, less degradation occurs before 26 hours for NPs synthesized at higher temperatures. Additionally, the slope of the first degradation step for low temperatures is slightly steeper, which indicates a higher degradation rate before 26 hours. As the slopes decrease after the first degradation stage (i.e., after 26 hours), the degradation rates of both types of MSNPs decrease. Finally, the degradation period for MSNPs synthesized at $65^{\circ} \mathrm{C}$ was 48 hours and for MSNPs synthesized at $80^{\circ} \mathrm{C} 70$ hours.

Following the findings of Yamada et al. [7], we do not expect the difference in degradation behavior to be due to the size differences of the NPs. Moreover, the zeta potential of the NPs did not change significantly upon varying the synthesis temperature. Therefore, the different NPs had a similar stability and we do not expect different aggregation behavior of the two samples. Hence, the degradation behavior was linked to the formation kinetics of the MSNPs. A lower synthesis temperature induces faster NP synthesis and therefore increases the chance of defect formation. Hence, the particle will be more prone to degradation.

Like the temperature variations, base concentration variations caused a clear tendency in the biodegradation kinetics, as illustrated in Figure 8. The degradation duration is longer for MSNPs produced with a larger amount of TEA. When $0.2 \mathrm{~g}$ TEA was used during synthesis, the MSNPs 

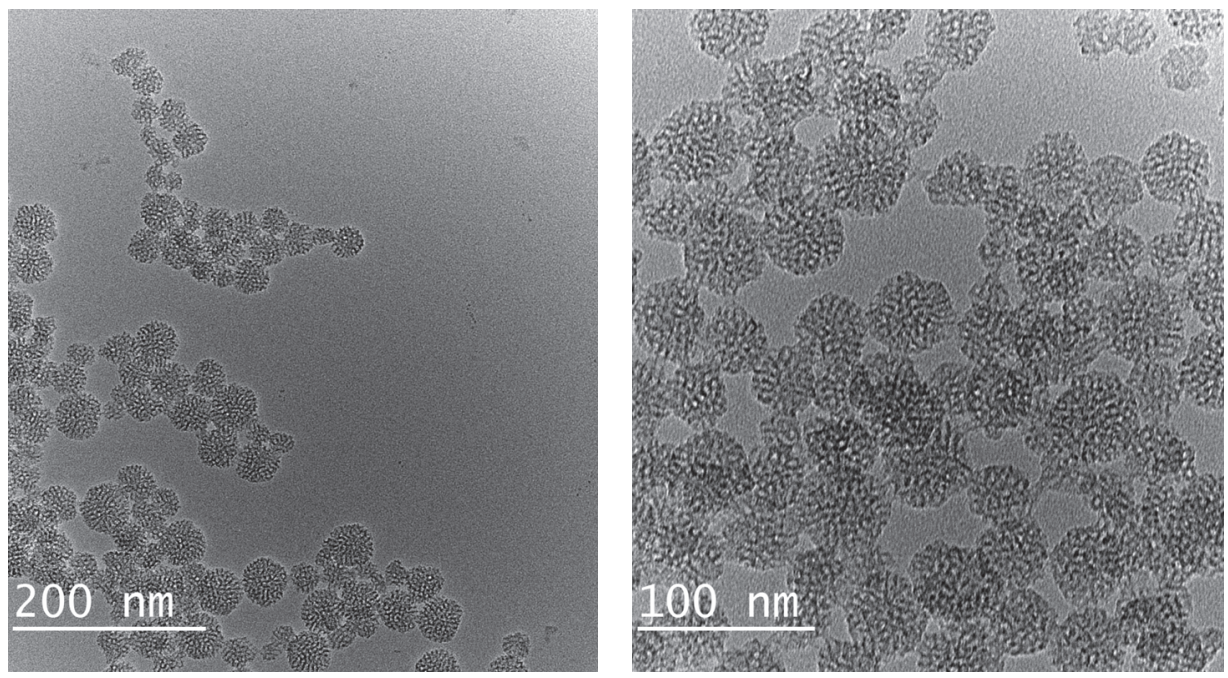

Figure 5: Typical TEM images of MSNPs.

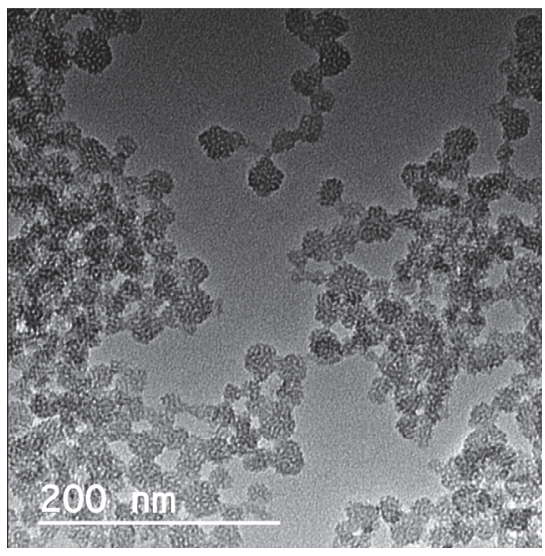

(a)

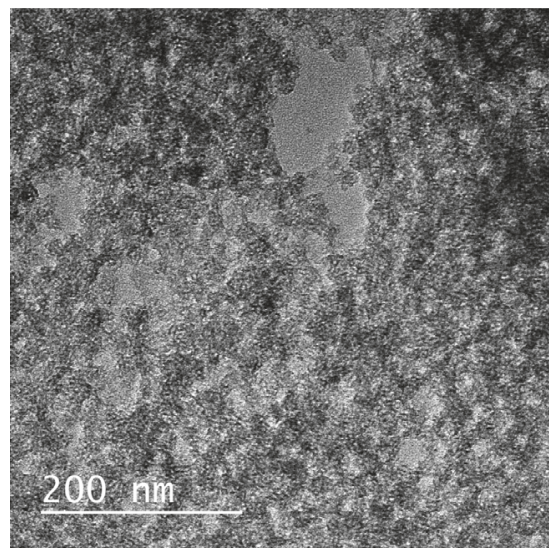

(b)

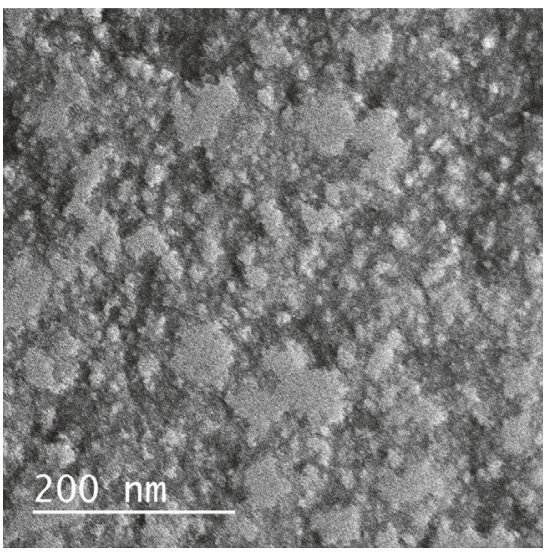

(c)

FIGURE 6: TEM images of degrading MSNPs. From (a) to (c): before degradation, after eight hours and twenty-four hours of degradation in PBS.

degraded over a period of 30 hours. If $0.8 \mathrm{~g}$ was used, this period increased fourfold to 120 hours. Considering the degradation rate, all conditions follow the same trend during the first few hours of the experiment. Afterwards, the degradation rate of NPs produced with the highest amount of TEA (MSNP-OH-4; 0.8 g TEA) deviates first from this linear behavior, followed by MSNP-OH-2 (0.4 g TEA). This deviation indicates decreased degradation rates for MSNPs produced with higher amounts of TEA, as reflected by the slopes of the respective curves in Figure 8. Large variations in electrochemical stability (zeta potential results) were observed; however, these are not consistent with the degradation data. Therefore, the growth rate of the particles determined the degradation behavior. NPs produced with high amounts of TEA were formed slowly due to the chelating effect of TEA, whereas NPs produced with low amounts of TEA were formed more rapidly, resulting in lower and higher biodegradability, respectively.
Another variable synthesis parameter in this work is the surface functionalization of the MSNPs. Figure 9 shows the degradation behavior of amine-functionalized MSNPs, carboxylated MSNPs, and MSNPs without functionalization (silanol coated). Both amine- and carboxylfunctionalized MSNPs degrade faster than nonfunctionalized MSNPs, and MSNPs with amine functionalization showed the fastest degradation behavior. This tendency is partially induced by the electrostatic stability of the particles. Aminefunctionalized and carboxylated NPs have a significantly larger zeta potential compared to nonfunctionalized MSNPs, indicating a higher electrostatic stability. Therefore, nonfunctionalized NPs have a higher chance to agglomerate during degradation, resulting in a decreased surface area available for the phosphate groups to degrade the particles, which explains the different degradation behavior of functionalized and nonfunctionalized MSNPs. However, the zeta potential of both amine and carboxylic functionalized MSNPs is comparable. 


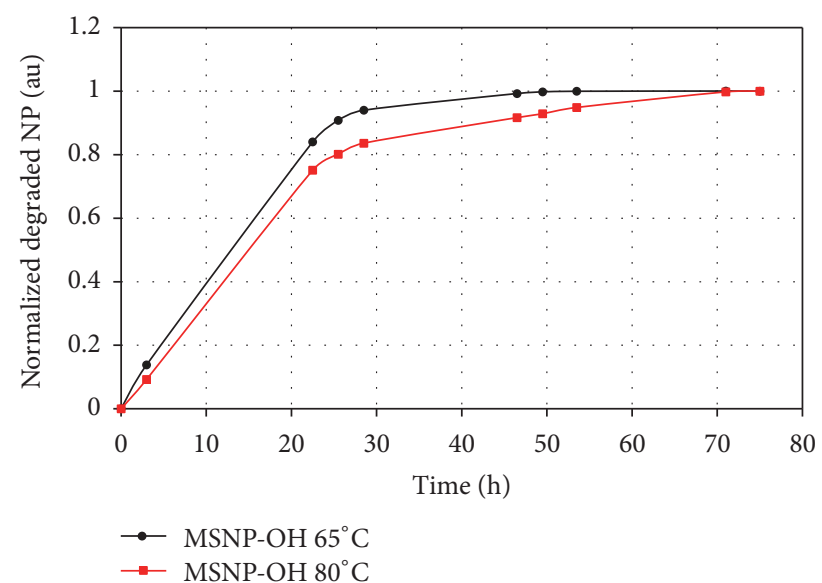

FIgURE 7: Degradation behavior of bare MSNPs synthesized at $65^{\circ} \mathrm{C}$ and $80^{\circ} \mathrm{C}$. NPs synthesized at $80^{\circ} \mathrm{C}$ degrade slower than NPs synthesized at $65^{\circ} \mathrm{C}$.

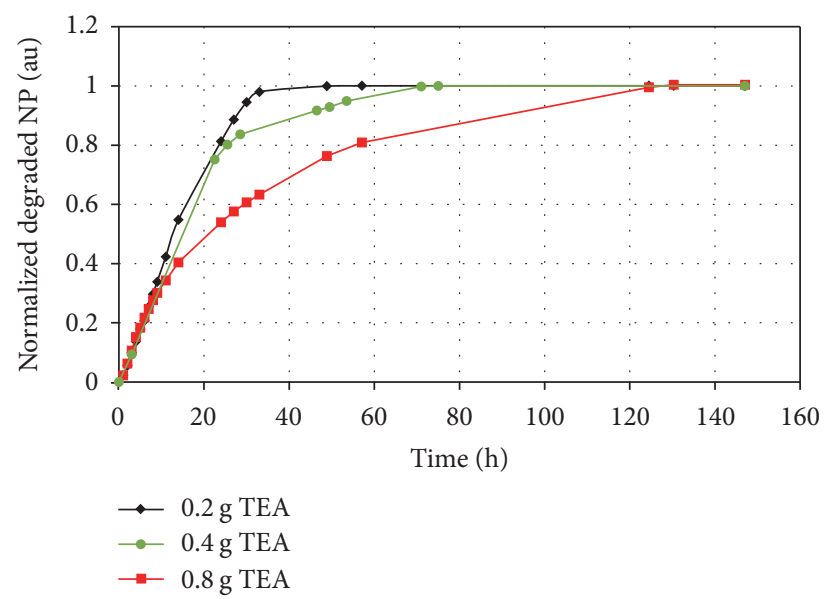

FIGURE 8: Degradation behavior of MSNPs synthesized with 0.2, 0.4 , and $0.8 \mathrm{~g}$ TEA. The lower the amount of TEA, the faster the nanoparticles degraded.

Their different degradation behavior can be attributed to the electrostatic interaction between the negatively charged phosphates and the NPs. The negatively charged carboxyl groups repel the phosphates, decreasing the degradation rate significantly. Amine groups, on the other hand, are positively charged and therefore attract the phosphate groups, enhancing the degradation of the MSNPs.

Finally, the influence of the amount of MSNPs to PBS on the degradation behavior was examined. The results, shown in Figure 10, indicate that the relative amount of MSNPs to PBS does not have a significant effect on the total degradation time, as the degradation end point remains the same for all conditions. However, fast degradation is observed very early in the experiment for low amounts of MSNPs (i.e., before the deviation of the linear behavior at 10 hours). The slope of the degradation curve of high amounts of MSNPs is clearly less steep before its deviation point, indicating slower degradation at the beginning of the experiment. To conclude, a high

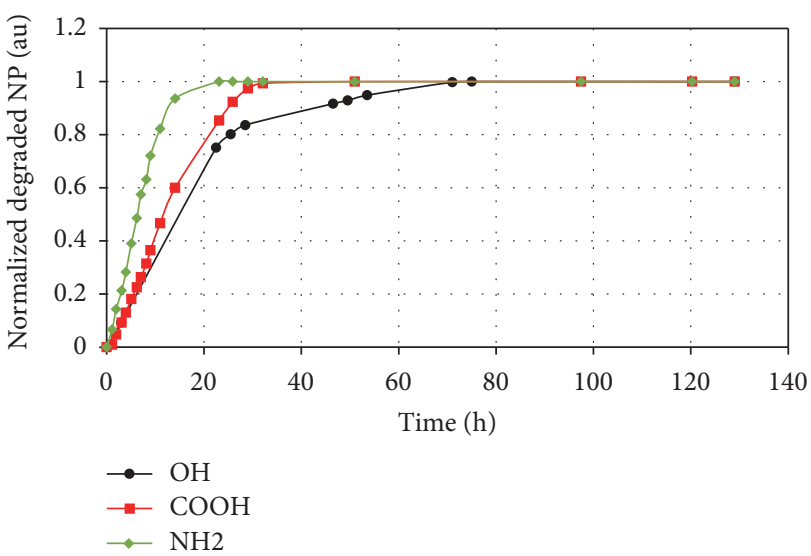

FIGURE 9: Degradation behavior of amine-, carboxyl-, and nonfunctionalized MSNPs. Amine-functionalized particles appear to be the most prone to degradation, followed by carboxylated and silanol functionalized NPs.

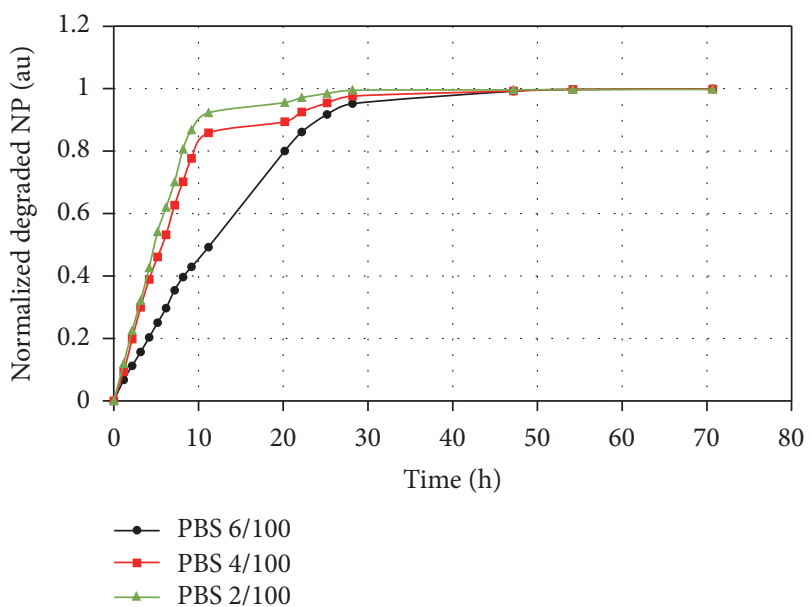

FIGURE 10: The effect of the amount of NPs on the biodegradation kinetics. The higher the amount of MSNPs, the slower degradation occurring.

relative amount of MSNPs has a slower first degradation step, preserving their structure significantly longer as compared to a low relative amount of MSNPs. High amounts of MSNPs show a faster second stage degradation step, eventually resulting in an equal end point of degradation for all conditions.

Since $80 \%$ of a particle degrades in the first degradation step (i.e., before the deviation from the linear behavior of the curve), we consider this first step to be the most important one. When keeping the NP concentration constant but increasing the NP amount, larger agglomerates can be formed, decreasing the degradation rate of larger amounts of particles. Note that the degradation curves display a normalized degradation behavior (i.e., the amount of degraded material divided by the total amount of degraded material at the end of the experiment). Therefore, the degradation results for different amounts of NPs are counterintuitive as we would expect the degradation behavior to depend on the concentration and not on the amount. 


\section{Conclusion}

In this work, the influence of synthesis and experimental parameters on the morphology and biodegradability of MSNPs was investigated by means of an optimized molybdenum blue chemistry and spectrophotometric analysis. Additionally, it was shown that the degradation behavior, as well as the NP characteristics, could easily be adjusted by small changes in the relatively simple synthesis procedure. The variable synthesis parameters were temperature, concentration of catalyst, and surface functionalization of MSNPs. The obtained results regarding NP characteristics were comparable to earlier publications of Möller et al. [24]. The NPs were found to be smaller when synthesized at lower temperatures or with low amounts of TEA, and this effect was due to the chelating properties of TEA. Regarding the degradation behavior, we observed a two-step degradation behavior for all conditions as opposed to the results of Yamada et al. [7] and $\mathrm{He}$ et al. [6] who observed a onestep and three-step degradation behavior, respectively. This difference is due to the higher amounts of NPs used by Yamada et al., explaining their slow degradation and the lower concentrations used by $\mathrm{He}$ et al.

Upon increasing synthesis temperature and increasing amount of TEA, the degradation duration extended whilst the degradation rate decreased. Regarding surface functionalization, the degradation kinetics were the slowest for nonfunctionalized, followed by carboxylated and aminofunctionalized MSNPs. The degradation behaviors of functionalized MSNPs might be of interest when further binding of molecules is necessary for the application (e.g., drugs, proteins, and RNA). Finally, the degradation was influenced by the amount of MSNPs in PBS. A lower NP amount resulted in a fast first degradation step, followed by a slow second step. On the other hand, a high amount of MSNPs showed a slow first degradation step, compared to low amounts of MSNPs. The duration of degradation remained unchanged. This phenomenon should be considered to set the right dose for drug delivery applications or can be used for drug release at specific targeted sites in the body.

Based on our results, we believe the NP degradation to be strongly dependent on the formation kinetics of the NPs during synthesis and the charge of the coating layer. In this regard, fast condensation (i.e., low amount of TEA or low synthesis temperatures) could result in a higher chance of defect formation, lowering the structural stability of the particles. Additionally, positively charged surface functionalization (such as amine functionalization) enhances degradation behavior, due to the attractive electrostatic interaction between the amine groups of the NPs and the phosphate groups of PBS. Therefore, low synthesis temperatures, low amounts of TEA, and amine functionalization give rise to particles which undergo fast biodegradation in PBS.

\section{Conflicts of Interest}

The authors declare that there are no conflicts of interest regarding the publication of this article.

\section{Authors' Contributions}

Stephanie Seré and Bert De Roo contributed equally to this work.

\section{Acknowledgments}

The authors want to express their gratitude to the Research Foundation-Flanders and the Agency for Innovation by Science and Technology in Flanders for their financial support (Stephanie Seré Grant no. 1S52016N and Bert De Roo Grant no. IWT/121661). The authors thank the EU for funding via the FP7 Grant CosmoPHOS-nano Project (Project no. 310337). Jin Won Seo acknowledges the financial support from the Hercules Foundation (Project no. HER/08/25). The authors acknowledge the Olivia Fund for their financial support. Finally, they also thank Carmen Bartic and Olivier Deschaume for the use of the spectrophotometer.

\section{References}

[1] Y. Lu, H. Fan, A. Stump, T. L. Ward, T. Rieker, and C. J. Brinker, "Aerosol-assisted self-assembly of mesostructured spherical nanoparticles," Nature, vol. 398, no. 6724, pp. 223-226, 1999.

[2] S. H. Joo, J. Y. Park, C.-K. Tsung, Y. Yamada, P. Yang, and G. A. Somorjai, "Thermally stable Pt/mesoporous silica core-shell nanocatalysts for high-temperature reactions," Nature Materials, vol. 8, no. 2, pp. 126-131, 2009.

[3] V. Biju, "Chemical modifications and bioconjugate reactions of nanomaterials for sensing, imaging, drug delivery and therapy," Chemical Society Reviews, vol. 43, no. 3, 2014.

[4] I. I. Slowing, B. G. Trewyn, S. Giri, and V. S.-Y. Lin, Advanced Functional Materials, vol. 17, 8, 2007.

[5] Z. Li, J. C. Barnes, A. Bosoy, J. F. Stoddart, and J. I. Zink, "Mesoporous silica nanoparticles in biomedical applications," Chemical Society Reviews, vol. 41, no. 7, pp. 2590-2605, 2012.

[6] Q. He, J. Shi, M. Zhu, Y. Chen, and F. Chen, “The three-stage in vitro degradation behavior of mesoporous silica in simulated body fluid," Microporous and Mesoporous Materials, vol. 131, no. 1-3, pp. 314-320, 2010.

[7] H. Yamada, C. Urata, Y. Aoyama, S. Osada, Y. Yamauchi, and K. Kuroda, "Preparation of colloidal mesoporous silica nanoparticles with different diameters and their unique degradation behavior in static aqueous systems," Chemistry of Materials, vol. 24, no. 8, pp. 1462-1471, 2012.

[8] D. Napierska, L. C. J. Thomassen, V. Rabolli et al., "Sizedependent cytotoxicity of monodisperse silica nanoparticles in human endothelial cells," Small, vol. 5, no. 7, pp. 846-853, 2009.

[9] V. Rabolli, L. C. J. Thomassen, C. Princen et al., "Influence of size, surface area and microporosity on the in vitro cytotoxic activity of amorphous silica nanoparticles in different cell types," Nanotoxicology, vol. 4, no. 3, pp. 307-318, 2010.

[10] L. C. J. Thomassen, A. Aerts, V. Rabolli et al., "Synthesis and characterization of stable monodisperse silica nanoparticle sols for in vitro cytotoxicity testing," Langmuir, vol. 26, no. 1, pp. 328-335, 2010.

[11] D. Napierska, L. C. J. Thomassen, D. Lison, J. A. Martens, and P. H. Hoet, "The nanosilica hazard: another variable entity," Particle and Fibre Toxicology, vol. 7, article 39, 2010. 
[12] Y.-S. Lin, N. Abadeer, and C. L. Haynes, "Stability of small mesoporous silica nanoparticles in biological media," Journal of the Chemical Society, vol. 47, no. 1, 2011.

[13] V. Cauda, C. Argyo, and T. Bein, "Impact of different PEGylation patterns on the long-term bio-stability of colloidal mesoporous silica nanoparticles," Journal of Materials Chemistry, vol. 20, no. 39, pp. 8693-8699, 2010.

[14] V. Cauda, A. Schlossbauer, and T. Bein, "Bio-degradation study of colloidal mesoporous silica nanoparticles: Effect of surface functionalization with organo-silanes and poly(ethylene glycol)," Microporous and Mesoporous Materials, vol. 132, no. 1-2, pp. 60-71, 2010.

[15] N. K. Hon, Z. Shaposhnik, E. D. Diebold, F. Tamanoi, and B. Jalali, "Tailoring the biodegradability of porous silicon nanoparticles," Journal of Biomedical Materials Research Part A, vol. 100, no. 12, pp. 3416-3421, 2012.

[16] W. S. Rasband, “ImageJ. U. S," 2016, http://imagej.nih.gov/ij/.

[17] C. M. Dekeyser, C. C. Buron, S. R. Derclaye, A. M. Jonas, J. Marchand-Brynaert, and P. G. Rouxhet, "Degradation of bare and silanized silicon wafer surfaces by constituents of biological fluids," Journal of Colloid and Interface Science, vol. 378, no. 1, pp. 77-82, 2012.

[18] H. Yang, C. Li, C. Wei et al., "Molybdenum blue photometry method for the determination of colloidal silica and soluble silica in leaching solution," Analytical Methods, vol. 7, no. 13, pp. 5462-5467, 2015.

[19] T. Coradin, D. Eglin, and J. Livage, "The silicomolybdic acid spectrophotometric method and its application to silicate/biopolymer interaction studies," Spectroscopy: An International Journal, vol. 18, no. 4, pp. 567-576, 2004.

[20] J. D. H. Strickland, "The preparation and properties of silicomolybdic acid. I. The properties of alpha silicomolybdic acid," Journal of the American Chemical Society, vol. 74, no. 4, pp. 862$867,1952$.

[21] J. D. H. Strickland, "The preparation and properties of silicomolybdic acid. II. The preparation and properties of $\beta$-silicomolybdic acid," Journal of the American Chemical Society, vol. 74, no. 4, pp. 868-871, 1952.

[22] J. D. H. Strickland, "The preparation and properties of silicomolybdic acid. III. The combination of silicate and molybdate," Journal of the American Chemical Society, vol. 74, no. 4, pp. 872$876,1952$.

[23] C. X. Galhardo and J. C. Masini, "Spectrophotometric determination of phosphate and silicate by sequential injection using molybdenum blue chemistry," Analytica Chimica Acta, vol. 417, no. 2, pp. 191-200, 2000.

[24] K. Möller, J. Kobler, and T. Bein, "Colloidal suspensions of nanometer-sized mesoporous silica," Advanced Functional Materials, vol. 17, no. 4, 2007. 


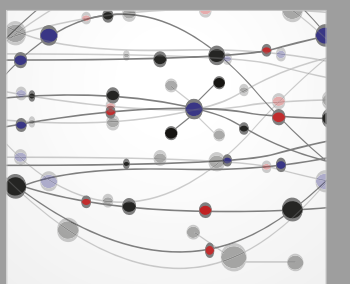

The Scientific World Journal
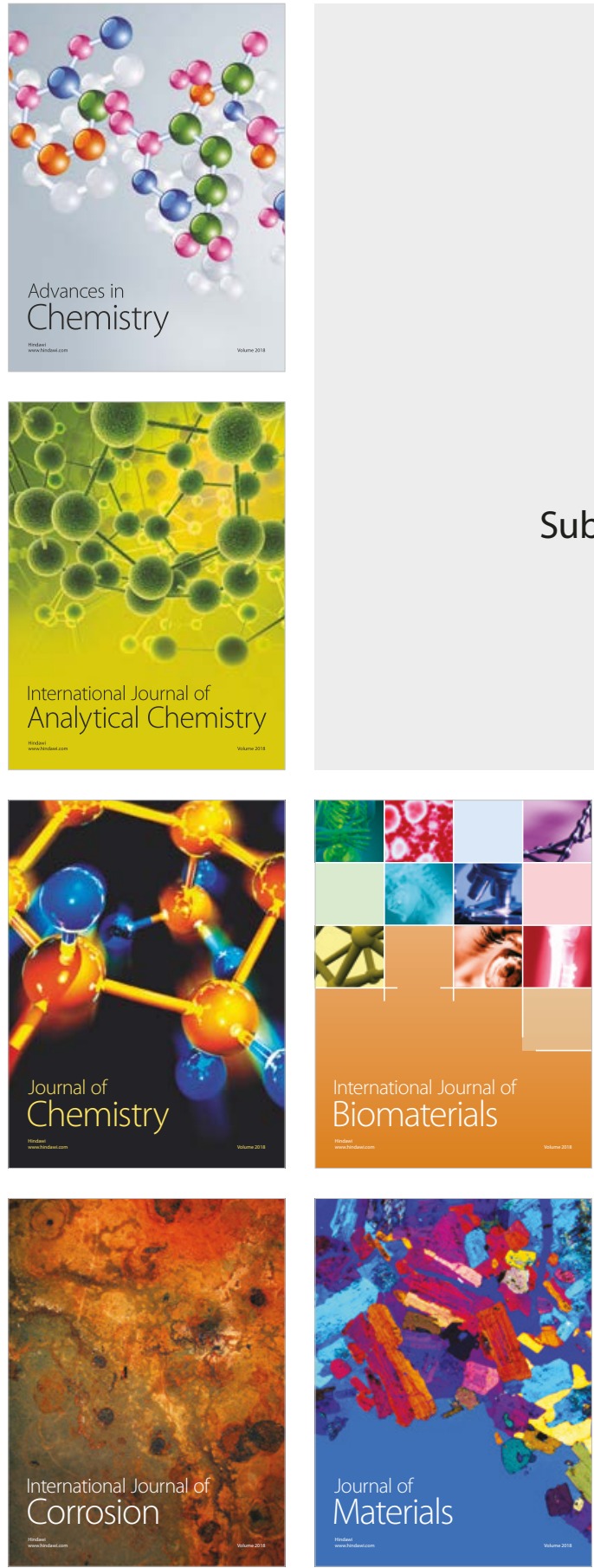

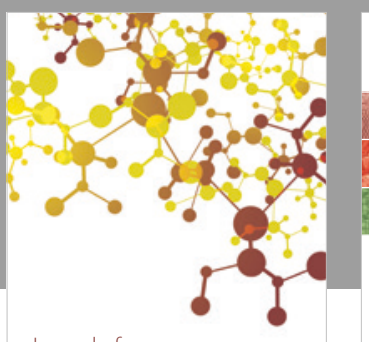

Journal of

Applied Chemistry
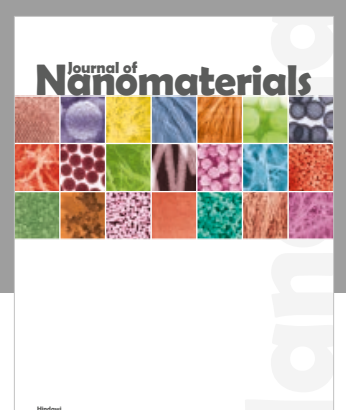

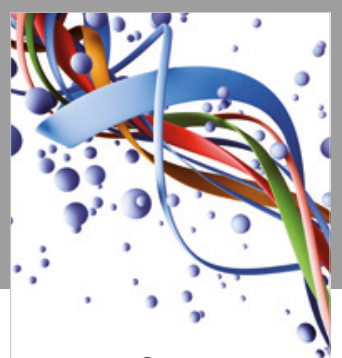

Scientifica

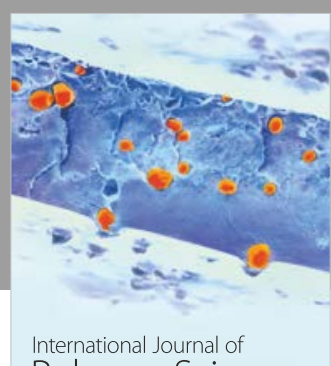

Polymer Science

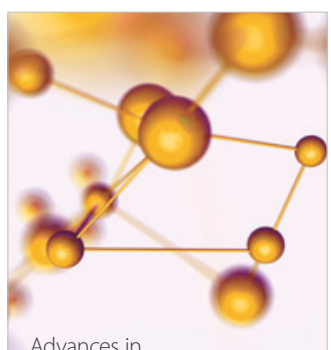

Physical Chemistry
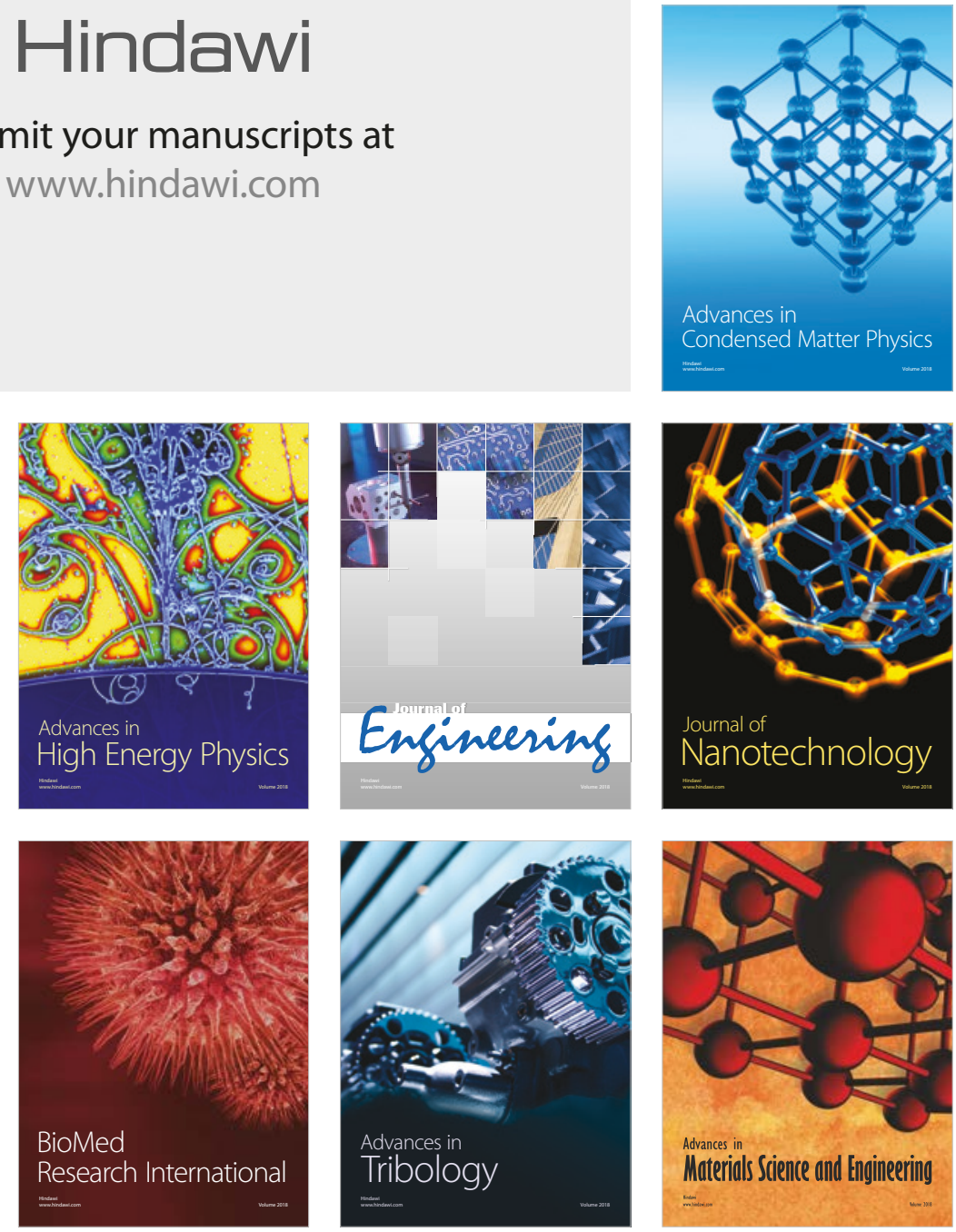